\title{
GSU Praat Tools: Scripts for modifying and analyzing sounds using Praat acoustics software
}

\author{
Michael J. OWREN \\ Georgia State University, Atlanta, Georgia
}

\begin{abstract}
The Praat acoustics program (Boersma, 2001) is powerful freeware that is widely used by behavioral scientists working with digital sound. This article describes GSU Praat Tools, a script package that helps simplify and automate such work. The routines use Praat's scripting language to create new menus and commands within the existing interface, and can operate either on individual files or in batch mode. The new functions facilitate selecting, displaying, editing, filtering, and otherwise modifying sounds, quantifying acoustic features, and saving results in text-based data files. The package includes an installation script and user's manual, and is available free from psyvoso.googlepages.com/softwaredownload.
\end{abstract}

The digital revolution has provided many benefits for the behavioral sciences, not the least of which have been in audio technology. Advances in this area have, for instance, made it much easier to record, modify, analyze, and synthesize sounds, as well as to use sounds in experiments. Furthermore, whereas there were once only a few programs available for working with digital sound, now there are many. The software includes a wide range of commercial, shareware, and freeware programs, offering a diversity of capabilities and features. As is often the case, however, this software exhibits a general tradeoff between ease of use on the one hand and versatility and power on the other (see zeeman.ehc.edu/envs/Hopp/ sound.html for listings of a number of acoustics programs and related resources).

At the simpler end of things are programs such as Spectrogram (Visualization Software LLC), which digitizes sounds, presents high-quality graphical representations of waveforms, individual spectra, and time-by-frequency spectrograms, allows cursor-based measurements, and is practically effortless to learn. Raven (Cornell Lab of Ornithology) occupies an intermediate level in providing elegant graphical displays and straightforward measurement of sound features, but also demands some study and effort by the user. The built-in measures are limited, focusing on basic time, amplitude, and frequency characteristics. SpeechStation2 (Sensimetrics Corporation) is similar in requiring some learning while providing a useful but smallish set of measures. Useful functionalities such as pitch tracking and resonance modeling are included in this program; graphics are simple and relatively inflexible.

The more powerful and flexible programs are those that, for example, provide direct access to the sound data, a variety of analysis and synthesis algorithms, and scripting capabilities for creating custom functions. The inher- ent flexibility involved demands more of the user, not only in order to take full advantage of program capabilities but also in getting started in relatively simple tasks. The Praat acoustics software (Boersma, 2001) falls into this category, even though it includes a graphical interface with pull-down menus (Engineering Design's Signal software is another example). Praat provides so many menus, commands, and parameter settings that the novice user can feel quite overwhelmed. There are currently, for instance, 13 different "editor" windows through which to visualize and modify critical sound features, five variants of the pitch-extraction command, four different linear predictive coding (LPC) algorithms, and almost 90 user-accessible mathematical functions.

The reward for mastery is nonetheless great, since Praat is very powerful (but open-source) freeware, available from www.praat.org, that runs on a diverse set of platforms (including Macintosh, Windows, Linux, FreeBSD, SGI, Solaris, and HP-UX), and is updated at an astonishing clip by its authors, Paul Boersma and David Weeninck. This compelling combination has made Praat quite popular in speech science, and the program is rapidly finding new users among bioacousticians and others as well. Furthermore, even a cursory Internet search reveals a number of resources for Praat from other sources, including customwritten Praat scripts and an active users' group (uk.groups .yahoo.com/group/praat-users). Whereas most of the scripts are designed as more or less stand-alone routines that perform narrowly conceived tasks, more-integrated script packages are available. One example is Akustyk (bartus .org/akustyk), which provides a comprehensive toolbox for use in analyzing and synthesizing vowel sounds. This article introduces another integrated set of scripts, dubbed GSU Praat Tools. These routines are designed to simplify and automate a number of the basic operations that behav-

M. J. Owren, owren@gsu.edu 
ioral scientists might want to perform with Praat, and can be useful with virtually any kind of sound. The following sections provide a basic description of the system, as well as some examples of how it might be applied.

\section{OVERVIEW OF GSU PRAAT TOOLS}

GSU Praat Tools consists of text-based routines written in Praat's scripting language that are accessed through menus and commands added to the Praat graphical interface. It is designed to help Praat users explore a set of sounds of interest, to edit, filter, rescale, or otherwise modify these sounds to prepare them for or use them as experimental stimuli or for analysis, and to perform quantitative analyses whose results are sent to easily interpretable data files that can be read into spreadsheet or statistical programs. The routines support both interactive and more-automated data extraction, and can operate both on individual sound files and in batch mode. The scripts are meant to complement rather than to fundamentally change or extend Praat's native features, in many cases simply freeing the user from doing the nitty-gritty work of opening and closing files, creating displays, setting windows, and extracting quantitative data. While significantly speeding workflow, the scripts can also pause as needed at key points to allow the user to examine results and potentially intervene.

The code involved is generally simple, and has not been optimized for greatest efficiency. The emphasis is instead on uniformity and transparency. Because Praat scripts are simply text files that call the same commands found in the Praat menus, virtually any user can become proficient in writing them. Each GSU Praat Tools script therefore includes copious comments, in the hope that users will modify the routines to suit their own needs. On the other hand, no knowledge of scripting is needed in order to use this package - a given user may never even look at the underlying code.

\section{General Features}

Installation and user's manual. The GSU Praat Tools scripts have been tested on Mac OS X and Windows XP systems and, with the exception of the installation routine, are likely to function on other platforms as well. The installation script works simply by copying the files to a directory called plugin_GSUPraatTools in the Praat preferences directory, the location of which varies by platform. On start-up, Praat accesses a file called setup.praat in this plugin directory, which guides the creation of new menus and commands in the Praat interface and links them to their respective scripts. These links are created anew each time Praat is started, meaning that the added menus and commands can be removed simply by deleting the plugin directory (available as an "uninstall" option in the installation routine). Updating the Praat program itself does not require reinstalling GSU Praat Tools. The distribution includes a manual in PDF format, as well as two sounds and corresponding label files that are meant to illustrate various features of the package. This article provides some information along these lines, but much more is available in the manual.

"Utility" versus "Analysis." GSU Praat Tools scripts fall into two basic categories, namely "Utility" and "Anal- ysis" routines. Utility scripts help automate routine fileprocessing operations; Analysis scripts conduct numerical analyses and write the results to text files. Scripts are thus invoked through commands found either in the GSU Analysis Tools or GSU Utility Tools menu (see Figure 1B). Other script-linked commands also appear in various menus, for instance, allowing multiple files to be read in or saved automatically, and creating one-click file navigation.

"Editor" versus "Objects" modes. Many of the GSU Praat Tools scripts operate in conjunction with either a Praat Sound Editor or TextGrid Editor window, or from files selected in the Objects window with nothing else open. These "modes" of operation are selected internally to each script. In Editor mode, the user tends to be interacting with the script, for instance running it repeatedly on the same file and working on sound files one at a time. If quantifying vocal-tract resonances from a variable set of sounds, for instance, the user might be targeting the particular segments within a file where formant-tracking was working best. Here, careful, repeated inspection of formant tracks overlaid on a spectrogram in the Sound Editor would be warranted. In Objects mode, the user typically operates on multiple files, with the script running through them all one after the other. In this case, the user might select a series of sound files in the Objects window and perform a utility or analysis procedure on each in turn with few, if any, pauses or interruptions.

Selecting segments and setting windows. When applicable, each script also provides facilities for manual or automatic selection of the particular segment of interest. The possibilities include operating over the entire file, one or more labeled intervals within a file, a manually selected segment, or a fixed-duration "window" positioned in relation to the file or a labeled interval (discussed in the next section). In this last case, the window can be automatically placed relative to a cursor location, the midpoint, the maximum-amplitude point, the beginning, or the end.

\section{Input-Output}

The "Praat_Data" directory. GSU Praat Tools read and write sound, label, and data files from and to a specific directory placed on the user's desktop. This directory has to be named "Praat_Data," and is created either by the user or as needed by the Analysis routines. All the scripts that read or write do so using that particular directory, mainly as a programming convenience.

Reading and writing multiple files. Commands added to the New menu of the Praat Objects window can be used to read from or write to a Praat Tools Project file, or to read or write multiple sound and label files. Project files are created using Praat's archiving command to bundle sound and label files present in the Objects window into a single file, along with any data files found in Praat_Data. Conversely, reading a project file adds the sound and label files it contains to the Objects window, while placing data files in Praat_Data. The Project function can thus be used to bundle and later recover all the critical files from a particular undertaking with a single command, which can be useful for both short- and long-term storage.

Data files. Data produced by analysis scripts can both be shown to the user in a Praat information window and 
A

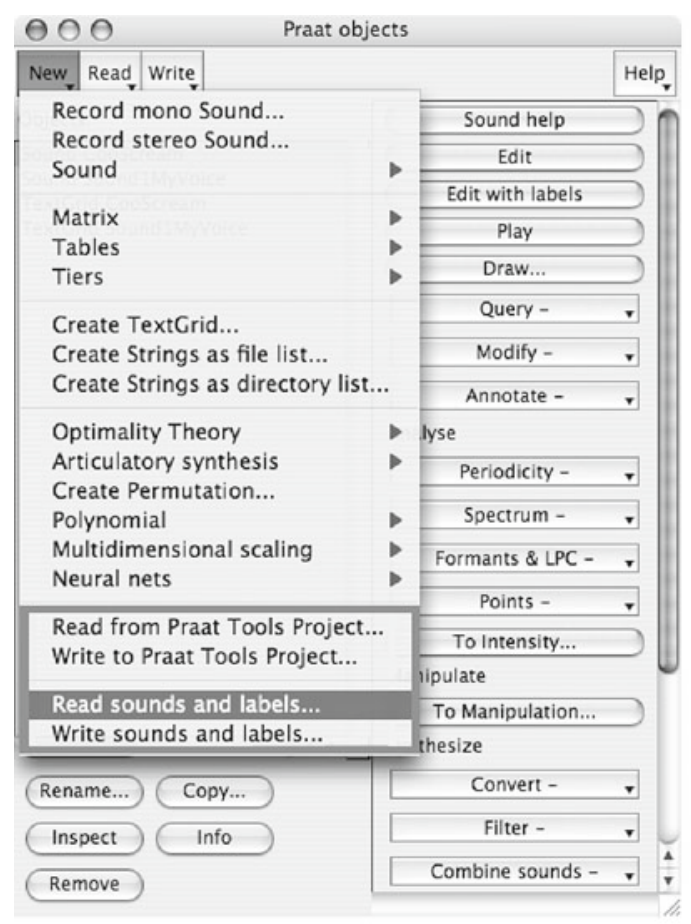

B

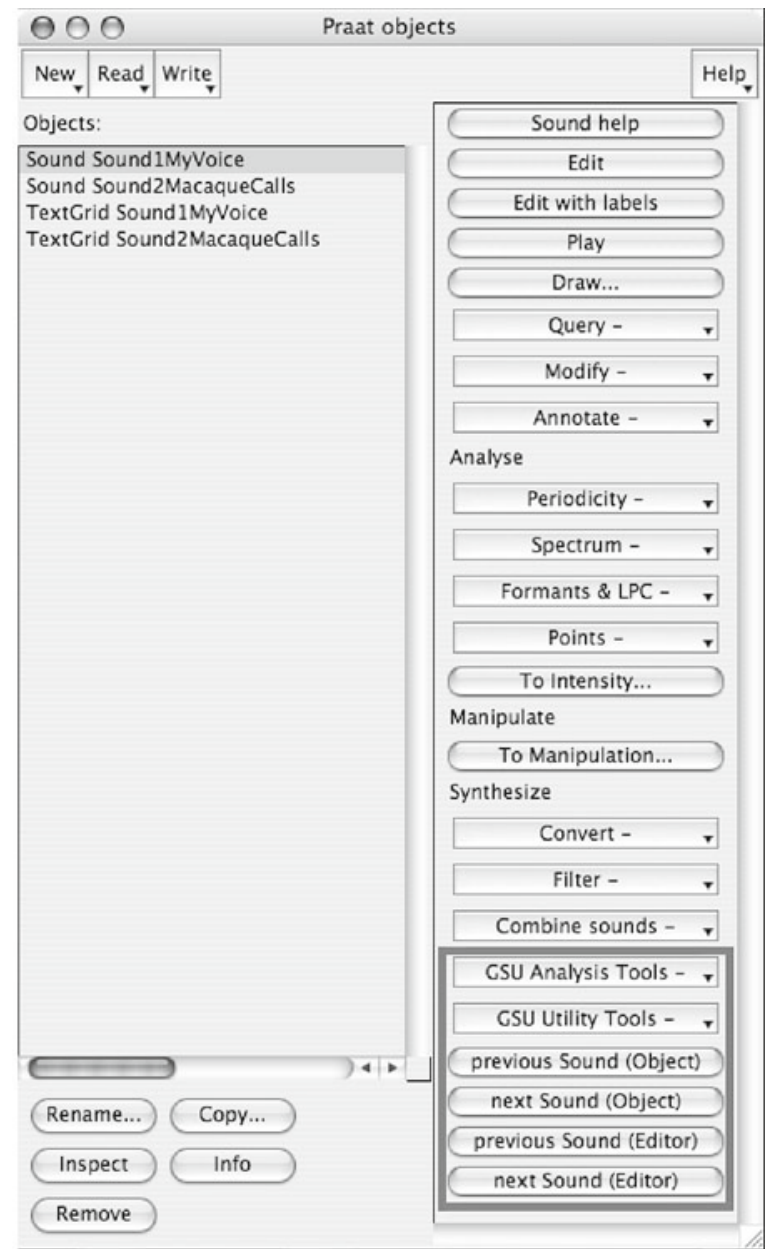

Figure 1. Location of GSU Praat Tools commands in the Praat Objects window. (A) File-reading and file-writing commands. (B) Menus and file-selection commands.

be appended to a text-based data file in Praat_Data (illustrated in Figure 2). Data files are automatically named for the scripts that produced them (with a ".out" extension), and are designed to be read into a spreadsheet or statistics program for further analysis. Performing several analyses on the same set of sound (and label) files can thus produce multiple data files with corresponding row order. Once in a spreadsheet, the various results from each file can be aligned through simple cut-and-paste operations to create a single, integrated data block.

All the ".out" files present in Praat_Data are included when a new Project file is created, but without disturbing the original versions in the directory. In order to help avoid inadvertently overwriting previous data, Analysis scripts always append to, rather than overwrite information in their respective data files. However, when data files are extracted from a Project file, the recovered data files will overwrite any data files already present in Praat_Data with the same name. The user is therefore advised to stay organized by archiving data files either in a Project file or under new, descriptive names as each analysis is completed and another is to begin.

\section{USING LABELS}

Praat works very well when labels are used to mark segments of interest within sound files. These labels are entered in Praat TextGrid files, which are specifically designed for this purpose. Although the labeling process itself can be a bit tedious, it allows automated analysis of the particular segments of interest within a file as well as providing identifiers that accompany the results sent to data files and greatly facilitate subsequent statistical comparisons. Although Praat does not place many constraints on label form, the GSU Praat Tools system does require that the user follow some specific conventions. Label appearance and use are illustrated in Figure 3.

Labeling itself is primarily done by hand using the Praat TextGrid Editor, with the script system providing some degree of automation. In GSU Praat Tools, a hierarchy of up to four levels of labeling can be used, each one corresponding to a Praat "Tier" within the TextGrid file. Up to four labels can be entered within each interval at each level, with no specific limit on how long an individual 
A

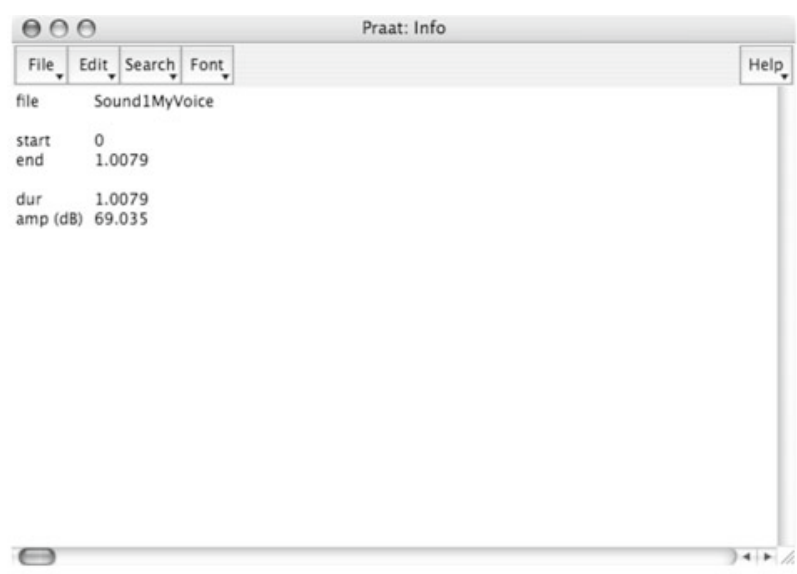

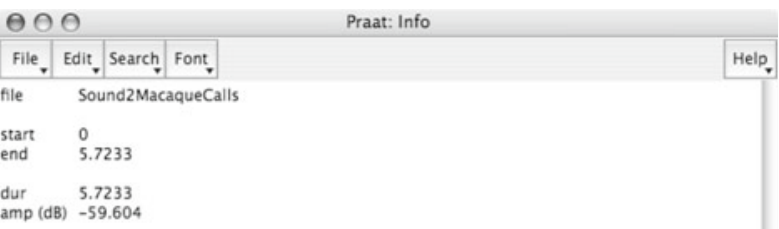

B

quantifyampdur.out

\begin{tabular}{|c|c|c|}
\hline$\odot \bigcirc 0$ & & quantifyampdur.out \\
\hline Mo/Day/ $\mathrm{Yr}$ Time & ProgramName & SoundName Begin End Duration Intensity (dB) \\
\hline Sep/7/2007 18:07:14 & quant ifyAmpDur & Sound1MyVoice 01.00791 .007969 .035 \\
\hline $\operatorname{Sep} / 7 / 200718: 07: 14$ & quant if yAmpDur & Sound2MacaqueCal Is o $5.7233 \quad 5.7233-59.604$ \\
\hline
\end{tabular}

Figure 2. Data generated by quantify Amp and Dur... in (A) the Praat information window and (B) the resulting quantifyampdur out file.

label can be. When data are extracted using either Editor (with labels) or Objects (with labels), the labels from each of the Tiers present are recovered and combined. At this stage of development, the scripts always start from the most fine-grained label level and work their way down. Multiple versions of the same TextGrid files, but with different numbers of Tiers, may thus be required for full analysis. Finally, because the user can choose to enter anywhere from one to four labels for each segment of interest, the scripts also send placeholder labels to the data file as needed to keep data columns aligned.

\section{OVERVIEW OF GSU PRAAT TOOLS COMMANDS}

This section reviews the commands and corresponding scripts that make up GSU Praat Tools. A complete list of command and script names is shown in Table 1.

\section{General Commands}

previous Sound, next Sound. These commands are found in corresponding forms in the Objects window, the Sound Editor, and the TextGrid Editor. In each case, file selection is moved backward or forward in the Object file sound list. If a Sound or TextGrid Editor is open, the previous and next commands close the editor, change the file selection, and then reopen the editor.
Edit with labels. If a sound file has been selected, this command opens a TextGrid editor for that file. If an identically named TextGrid file exists in the list and has not been selected along with the sound, both are selected before the editor opens. If no corresponding TextGrid exists, the script creates and opens it along with the sound.

\section{Utility Tools}

choose Window . . . This routine allows the user to select specific segments of interest within a sound in any of the ways enumerated in the earlier discussion. The script's parameter-entry form is meant to be left open on the screen when the user is working interactively-for instance, when exploring sounds one after another, but not yet conducting formal analyses.

display Spectrum . . . In order to facilitate exploration of sound features, as well as fast Fourier transform (FFT) and LPC analysis parameters, this command displays an FFT spectrum, an LPC-based smooth spectral envelope, or an LPC overlaid on a corresponding FFT.

edit Sound... This command allows the user to crop or "zeropad" a sound. To crop, the user either selects the segment to be retained, or slices off a fixed length from the beginning, end, or both ends of the sound. Zeropadding means that silent, zero-amplitude segments are added to the file, either at the beginning, at the end, or at both ends of the file. 
A

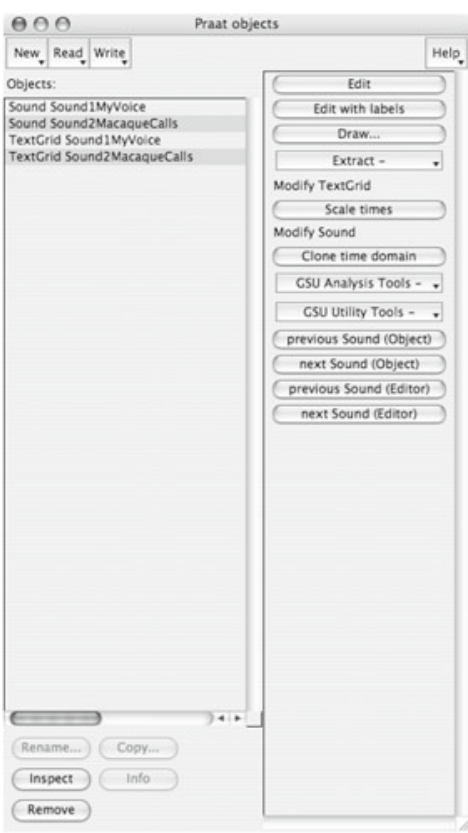

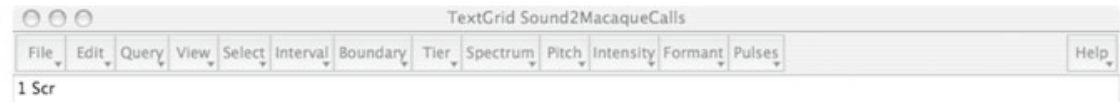
Scr

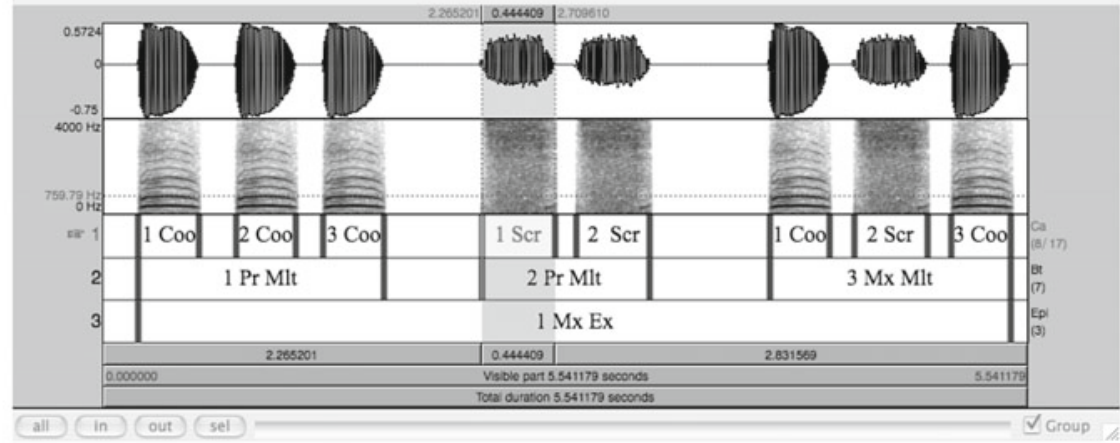

B

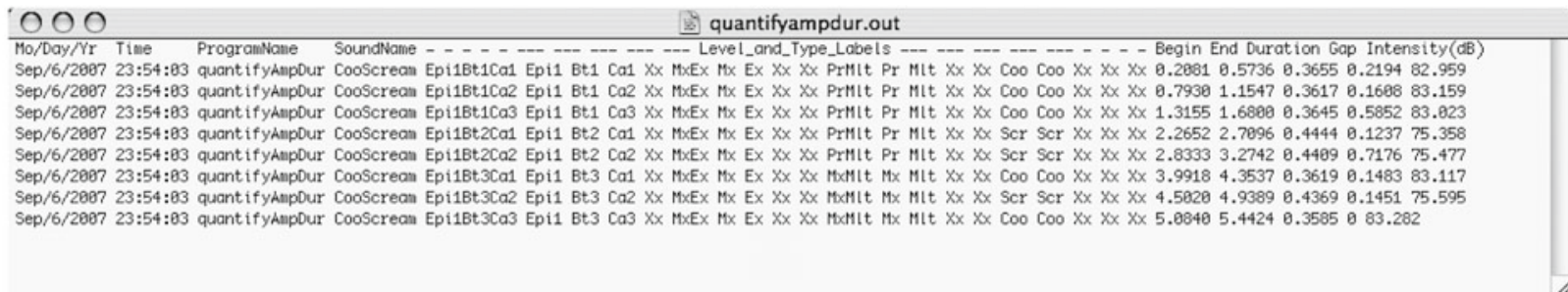

Figure 3. (A) A sound and label file opened in a TextGrid Editor. The calls have been labeled at three levels, including "call" (Ca), "bout" (Bt), and "episode" (Epi). Each interval also includes multiple type labels at each level. (B) The data file that results from running quantify Amp and Dur ... in Objects (with labels) mode on this particular pair of sound and label files.

event Detector . . . "Event detection" refers to finding the approximate onset and offset points for every sound segment occurring within a waveform file. These points are marked in an associated TextGrid file, with the determination that a sound has started or ended being based on user-specified amplitude thresholds and separation times. Whereas "misses," "false alarms," and other inaccuracies are common, the routine can nonetheless be a significant time-saver when labeling the segments of interest within a sound file.

modify Sound . . . This command rescales, filters, or adds noise to a sound. Rescaling can be multiplication by a scalar value, scaling by peak-amplitude value, or scaling by intensity $(\mathrm{dB})$. Filtering can be passband, stopband, or applying a moving-average smoothing window. Either Gaussian or uniform noise can be added, with the user controlling its relative amplitude.

preprocess Sound . . . The "Preprocessing" script includes four possible operations found to be consistently useful in preparing sounds as experimental stimuli or for analysis. These operations are removing "DC offset" (centering the sound waveform around 0 ), removing $60-\mathrm{Hz}$ energy (usually extraneous AC "hum"), rescaling sound amplitude to the full range that can be represented in the file (ensuring approximately equal loudness across files), and applying a linear amplitude ramp to the beginning, the end, or both ends of a file.

\section{Analysis Tools}

quantify Amp and Dur . . This routine is the simplest of the analysis scripts, focusing just on the amplitude and duration of a file, interval, or segment. When labeled intervals within a file are processed, the duration of the gap between adjacent intervals is also reported.

quantify FFT . . . In Editor mode, this command is used to interactively retrieve frequency and amplitude information for user-selected peaks in an FFT spectrum. It also calculates spectral slope and spectral moment values. In Objects mode, only slope and spectral moments are returned. 
Table 1

Listing of Commands Added to the Praat Menus, With Corresponding Script Names

\begin{tabular}{|c|c|c|}
\hline Script Type & Command Name & Script Name \\
\hline File navigation & $\begin{array}{l}\text { previous Sound (Objects) } \\
\text { next Sound (Objects) } \\
\text { previous Sound (Editor) } \\
\text { next Sound (Editor) } \\
\text { previous Sound (in Editor menu) } \\
\text { next Sound (in Editor menu) } \\
\text { previous Sound (in TextGrid menu) } \\
\text { next Sound (in TextGrid menu) }\end{array}$ & $\begin{array}{l}\text { previousObjects } \\
\text { nextObjects } \\
\text { previousEditor } \\
\text { nextEditor } \\
\text { previousSoundEditor } \\
\text { nextSoundEditor } \\
\text { previousTextGridEditor } \\
\text { nextObjectsTextGrid }\end{array}$ \\
\hline File reading and writing & $\begin{array}{l}\text { Read sounds and labels... } \\
\text { Write sounds and labels... } \\
\text { Read Praat Tools project. . . } \\
\text { Write Praat Tools project... }\end{array}$ & $\begin{array}{l}\text { readAllSoundsLabels } \\
\text { writeAllSoundsLabels } \\
\text { readPraatToolsProject } \\
\text { writePraatToolsProject }\end{array}$ \\
\hline Utility tools & $\begin{array}{l}\text { choose Window. . . } \\
\text { display Spectrum... } \\
\text { edit Sound... } \\
\text { event Detector. . . } \\
\text { modify Sound... } \\
\text { preprocess Sound... }\end{array}$ & $\begin{array}{l}\text { chooseWindow } \\
\text { displaySpectrum } \\
\text { editSound } \\
\text { eventDetector } \\
\text { modifySound } \\
\text { preprocessSound }\end{array}$ \\
\hline Analysis tools & $\begin{array}{l}\text { quantify Amp and Dur... } \\
\text { quantify FFT... } \\
\text { quantify Formants... } \\
\text { quantify LPC... } \\
\text { quantify Source... }\end{array}$ & $\begin{array}{l}\text { quantifyAmpDur } \\
\text { quantifyFFT } \\
\text { quantifyFormants } \\
\text { quantifyLPC } \\
\text { quantifySource }\end{array}$ \\
\hline
\end{tabular}

quantify Formants . . . In Editor mode, this script calculates mean frequencies and bandwidths from formant tracks displayed in a file, interval, or segment, as well as spectral slope and spectral moments. In Objects mode, only the latter are reported.

quantify $L P C \ldots$. . This routine is also designed for interactive peak-retrieval when used in Editor mode, in this case reporting frequency and amplitudes values from an LPC display, and computing spectral slope and spectral moments. In Objects mode, it only returns the latter.

quantify Source... This command computes a pitch track over a file, interval, or segment, showing the resulting contour in a Praat Pitch Editor. The user can then edit the track by either adding or removing points, and the script subsequently returns mean values for pitch, voicing, jitter, shimmer, and harmonicity.

\section{APPLICATION EXAMPLES}

\section{Example 1: Preparing Experimental Stimuli}

In this example, a set of sounds is prepared for use in a perceptual experiment by cropping, preprocessing, and zeropadding. The user must first copy the sound files into Praat Data, and when finished, should move them elsewhere.

1. Read the target sounds in from Praat_Data.

Command used: $\quad$ Read sounds and labels . .

Settings selected: Include user alerts

2. Preprocess the sounds by removing any DC offset, stopband filtering in the $60-\mathrm{Hz}$ region, and rescaling the entire sound by a factor that brings its highest-amplitude point to the maximum representable value. Scripts are run as a batch by selecting all the sound files in the Objects window and using Objects (no labels) mode.
Command used: $\quad$ preprocess Sound ...

Settings selected: $\quad$ Objects (no labels),

Remove DC offset,

Remove $60 \mathrm{~Hz}$,

Normalize amplitude

3. Crop each sound to isolate the specific segment of interest using Editor (no labels) mode. The user drags the cursor to mark the segment to retain, then runs the script. Cuts are made at the nearest zero-crossing points in the waveform, the editor closes, and the next sound is opened.

Command used: $\quad$ edit Sound...

Settings selected: $\quad$ Editor (no labels), Move on after, At zero crossings, Edit to make: crop

4. Add a 100-msec segment of silence at the beginning and end of each file to help avoid audible transients (e.g., clicks) when presented as experimental stimuli.

Command used:

edit Sound. . .

Settings selected: $\quad$ Objects (no labels), Edit to make: zeropad, Zeropad duration (msec): $100 \mathrm{msec}$,

Zeropad location: both ends

5. Save the sounds to the Praat_Data directory.

Command used: Write sounds and labels...

Settings selected: $\quad$ Select files: automatically (all) 


\section{Example 2: Sound Analysis Without Labels}

In this second example, a series of sounds is read in, preprocessed, and analyzed to determine each sound's amplitude, duration, pitch, perturbation, and formant structure. In addition, the frequency and amplitude of the first two harmonics in the Fourier spectrum are retrieved. Some operations are fully automated in batch mode, some can be executed in batch mode with pauses for user decisions at key points, and some are done by hand.

1. Sounds are read in using Read sound and labels ... .

2. Files are preprocessed using preprocess Sound ... to remove DC offset, filter out $60-\mathrm{Hz}$ energy, and perform amplitude normalization.

3 . Pitch and other source-energy information is extracted for each file in batch mode. The pitch-extraction process includes opening a Pitch Editor window and pausing for the user to inspect and edit pitch values. Pitch and vocal-perturbation data are shown in a Praat information window and sent to quantifysource.out.

Command used: quantify Source...

Settings selected:

$$
\begin{aligned}
& \text { Editor (no labels), } \\
& \text { Pause to check contour, } \\
& \text { Save data to file, } \\
& \text { Window placement: } \\
& \quad \text { entire file or interval }
\end{aligned}
$$

4. Formant-related information is extracted for a userselected segment of the sound. Each sound is viewed in spectrogram form in a Sound Editor. Segment boundaries are set at zero-crossings to avoid possible transients created by amplitude discontinuities.

Command used: quantify Formants ...

Settings selected:

$$
\begin{aligned}
& \text { Editor (no labels), } \\
& \text { Save data to file, } \\
& \text { Window placement: } \\
& \text { selected segment, } \\
& \text { Boundaries at zero crossings }
\end{aligned}
$$

5. Frequency and amplitude values are measured for the first two harmonics based on an FFT spectrum computed over a 1,024-point window centered on the maximumamplitude point of each file. The script finds that point and displays the corresponding FFT, then prompts the user to select each peak of interest in turn using the cursor. Values are shown in a Praat information window as each peak is retrieved, and both peak-related and other spectral information is sent to the data file quantifyfft.out in Praat_Data. (LPC-related information can be retrieved in similar fashion using the command quantify $L P C \ldots$, which saves data in quantifylpc.out.)

$\begin{array}{ll}\text { Command used: } & \text { quantify FFT... } \\ \text { Settings selected: } & \text { Editor (no labels), } \\ & \text { Save data to file, } \\ & \text { Maximum peaks to retrieve: 2, } \\ & \text { Window placement: } \\ & \text { around peak amplitude, } \\ & \text { Boundaries at zero crossings, } \\ & \text { Window in points: } 1024\end{array}$

6. Finally, the sounds and data files are saved as a single project file for archiving purposes. This file gets a name along the lines of 'myfirstproject"Today'sDate' .praattoolsProject.

Command used:

Write to Praat Tools Project...

Settings selected:

$$
\begin{aligned}
& \text { Project file name: } \\
& \text { myfirstproject, } \\
& \text { Select files: } \\
& \text { automatically (all), } \\
& \text { Include out files }
\end{aligned}
$$

\section{Example 3: Sound Analysis With Labels}

In a last example, sound, label, and data files are recovered from a previously created project file and analysis scripts are used to extract data from labeled segments within each file. Analyses are conducted entirely in Objects mode, TextGrid files with labels must be present in the Objects window, and each pair of sound and label files must have identical names. The scripts step through all the labeled intervals within each file, and then move on to the next file. Resulting data files now include label information for each analyzed segment (see Figure 3). The labels are tabulated both across and within levels, and are shown both in concatenated form and individually. In addition, placeholder labels $(\mathrm{Xx})$ are inserted as needed to equate the number of labels appearing in each row of data.

1. Sound, label, and data files are recovered from a project file using Read from Praat Tools Project ...

2. Amplitude and duration values are extracted over each labeled interval in each sound file selected in the Objects window. Data appear in an information window and are sent to the data file quantifyAmpDur.out as the script goes from interval to interval and file to file without pausing. A header line is sent to the data file at the beginning of the process, providing acronyms/abbreviations to remind the user what each value refers to.

Command used:

Settings selected:

$$
\text { quantify Amp and Dur... }
$$

$$
\begin{aligned}
& \text { Objects (with labels), } \\
& \text { Save data to file, } \\
& \text { Include data header, } \\
& \text { Window placement: } \\
& \text { entire file or interval }
\end{aligned}
$$

3. Pitch and other source-energy information is extracted for each labeled interval. Pitch extraction is restricted to the middle $60 \%$ of each segment, where automatic processing is likely to be the most reliable. In this case, the user chooses not to check each pitch contour as it is computed.

Command used:

Settings selected:

$$
\text { quantify Source... }
$$

Objects (with labels), Save data to file, Include data header, Window placement: entire file or interval, Percentage of current selection: 60 


\section{CONCLUSIONS}

The GSU Praat Tools package is available for free download from psyvoso.googlepages.com/softwaredownload, and includes a basic installation routine, a detailed user's manual, and two pairs of sound and label files that illustrate script function. The scripts are distributed under the provisions of the GNU General Public License, which explicitly encourages sharing and modification of software. The package is currently in version 1.4 , with future changes envisioned primarily as refinements to existing features and addition of new capabilities within the existing framework.

GSU Praat Tools is designed to help Praat users regardless of their level of expertise, but may also be useful to acoustics novices by inherently highlighting various preprocessing and analysis strategies that should be considered when examining a set of sounds. However, using the scripts effectively does depend on mastering the fundamentals of Praat, general acoustics, and vocal production. Links to tutorial material about Praat can be found on the Web site, and the users' group LISTSERV is a very useful resource. Help pages within Praat provide a wealth of information about both sound analysis and particular commands, including the operations that GSU Praat Tools draws on. Numerous sites on the World Wide Web explain the basics of physical, vocal, and speech acoustics, with personal experience suggesting that graphically based presentations are particularly helpful.

Finally, basic information about acoustic analysis of both human and nonhuman sounds is available from a variety of sources. Johnson (2003) and Kent and Read (2001), for example, provide introductory-level overviews of acoustics and speech production, as well as analysis and synthesis of speech sounds. Baken and Orlikoff (2000) review many of the same topics in the context of clinical applications, but also include more technical information about instrumentation and analysis algorithms, and review available data on normative acoustics in the speech of men, women, and children. Owren and Bachorowski (2007) present a tutorial on acoustic analysis in the context of extracting emotion-related features in speech, including common mistakes to avoid and other practical tips. Finally, an edited volume by Hopp, Owren, and Evans (1998) reviews many different topics related to nonhuman acoustic signals, including sound production, underwater sound, infrasound and ultrasound, digital acquisition and representation, filtering, and analysis.

\section{AUTHOR NOTE}

Script design benefited significantly from the insights of members of the Fall 2006 Sound Practicum graduate seminar, including Rebecca Antworth, Lisa Heimbauer, Ioana Latu, Michael Philipp, Joshua Plotnik, and Rebecca Snyder. I thank three anonymous reviewers for their helpful comments on a previous version of this work. Development of GSU Praat Tools was supported in part by NIMH Prime Award 1 R01 MH65317-01A2 Subaward 8402-15235-X, by the Center for Behavioral Neuroscience under the STC Program of the National Science Foundation under Agreement No. IBN-9876754, and by funds from the Department of Psychology, Georgia State University. Correspondence concerning this article should be addressed to M. J. Owren, Department of Psychology, Georgia State University, P.O. Box 5010, Atlanta, GA 30302-5010 (e-mail: owren@gsu.edu).

\section{REFERENCES}

BaKen, R. J., \& Orlikoff, R. F. (2000). Clinical measurement of speech and voice (2nd ed.). San Diego: Singular.

Boersma, P. (2001). Praat, a system for doing phonetics by computer. Glot International, 5, 341-345.

Hopp, S. L., Owren, M. J., \& Evans, C. S. (Eds.) (1998). Animal acoustic communication: Sound analysis and research methods. Berlin: Springer.

JoHnson, K. (2003). Acoustic and auditory phonetics (2nd ed.). Malden, MA: Blackwell.

KENT, R. D., \& READ, C. (2001). Acoustic analysis of speech (2nd ed.). San Diego: Singular.

OWren, M. J., \& Bachorowski, J.-A. (2007). Measuring emotionrelated vocal acoustics. In J. A. Coan \& J. J. B. Allen (Eds.), Handbook of emotion elicitation and assessment (pp. 239-266). Oxford: Oxford University Press.

(Manuscript received October 30, 2007; revision accepted for publication February 1, 2008.) 